Gut, 1969, 10, 6-12

\title{
Three-dimensional structure of the human small intestinal mucosa in health and disease
}

\author{
C. A. LOEHRY AND B. CREAMER \\ From the Gastrointestinal Laboratory, St Thomas' Hospital, London
}

Variations in the three-dimensional structure of the small intestinal mucosa have only been appreciated since the dissecting microscope was introduced as a tool for examining mucosal specimens (Rubin, Brandborg, Phelps, and Taylor, 1960). Although it is now widely accepted that the changes in mucosal structure from finger villi to leaves, convolutions, and a flat mucosa are all gradations of a single process, the reason for the mucosa adopting these morphological variations in disease states has remained largely obscure. Different authors have quoted a variety of possible mechanisms. Townley, Cass, and Anderson (1964) suggested that the primary mucosal defect was injury to the exposed upper parts of the villi causing denudation of the epithelium and basement membrane, and that these bare tips of villi 'fused', forming leaves and convolutions by 'bridging', producing a flat mucosa as progressively more of these villous clumps fused together. Himes and Adlersberg (1958) also suggested that epithelial cell injury caused villous fusion and clumping. Shiner and Doniach (1960), on the other hand, considered that the appearances could be the result of simple villous atrophy due to the "defective formation and viability of villous epithelial cells'. Swanson and Thomassen (1965) in a study of tropical sprue also subscribed to the idea of villous fusion, but considered that this began at the base of the villi rather than at the tips.

Previous studies of mucosal structure under the dissecting microscope have been limited in that only the upper part of the villi is directly visible, as the thick epithelial cell layer precludes any vision of the base of villi, of crypts, and of the crypt/villus relationship. In the present study the mucosa has been examined under the dissecting microscope after autolysis of epithelial cells, and histologically by serial horizontal cross sections through conventionally fixed specimens.

\section{METHODS}

AUTOLYSIS TECHNIQUE After death epithelial cells undergo rapid autolytic changes and can be washed away. The skeleton structure of the mucosa, however, remains, consisting of the basement membrane and connective tissue framework. These structures provide a true representation of the mucosal patterns present in vivo, and in addition allow direct vision of the crypt openings. The details of this technique have been previously described (Loehry and Creamer, 1966) and Fig. 1 shows the normal human jejunal mucosa before and after autolysis. There is no loss of villi by this technique, though their size is somewhat reduced after removal of the epithelial cell layer.

HORIZONTAL SERIAL SECTIONS In order to confirm the results obtained with the autolysed material and to demonstrate that the structures seen by this method did truly represent the mucosa in vivo, serial horizontal cross sections were taken down through fresh specimens of both normal and abnormal mucosa, and fixed in $10 \%$ formalin. Serial sections were taken at 10 to $15 \mu$ thickness from the tips of the villi to the crypt zone, stained with haematoxylin and eosin, and photographed. This method of examining the mucosa has also been employed by Cocco, Dohrmann, and Hendrix (1966), and by following down progressive sections the three-dimensional structure of the mucosa can be reconstituted.

\section{RESULTS}

Studied in these ways it becomes clear that the threedimensional structure of the small intestinal mucosa is more complex than has been thought. Over 200 specimens of mucosa were examined by the autolysed technique from necropsies so that a whole spectrum of variations in mucosal architecture in a large population was obtained. In these autolysed specimens structures became visible that had been previously obscured, revealing a smooth progression of changes in mucosal architecture from finger villi to a completely flat mucosa. For convenience of description the structural features of the mucosa are graded into five groups: stage 1, normal appearances in jejunum and ileum; stage 2, normal appearances in duodenum; stage 3 , mildly abnormal, characterized by broadened villi; stage 4 , moderately abnormal, characterized by convolutions; and 


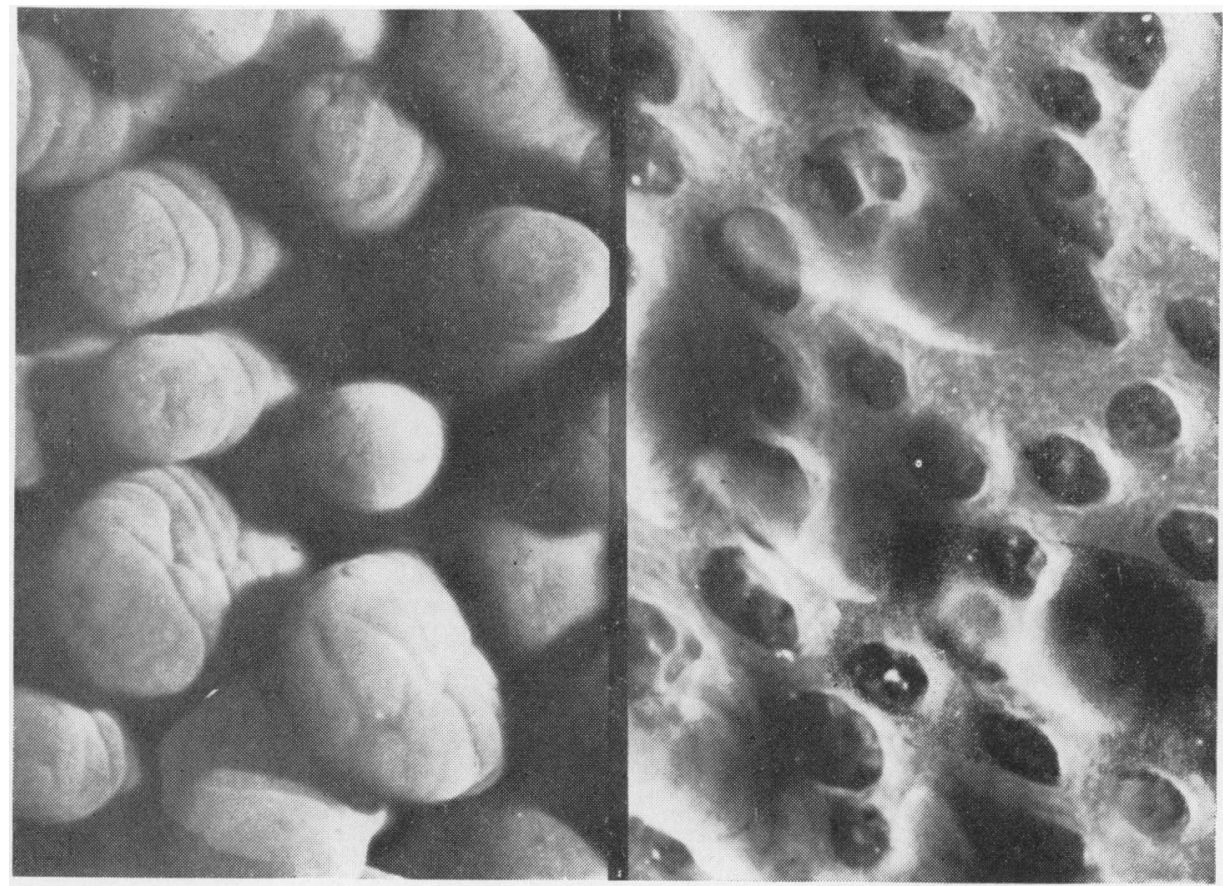

FIG. 1 .

Fig. 1. Human jejunal mucosa before and after autolysis. Removal of epithelial cells allows direct vision of the crypt openings. $\times 90$.

FIG. 2. Autolysed jejunal mucosa. Stage 1: the crypts outnumber villi in a ratio of approximately 3 to $1 . \times 90$.

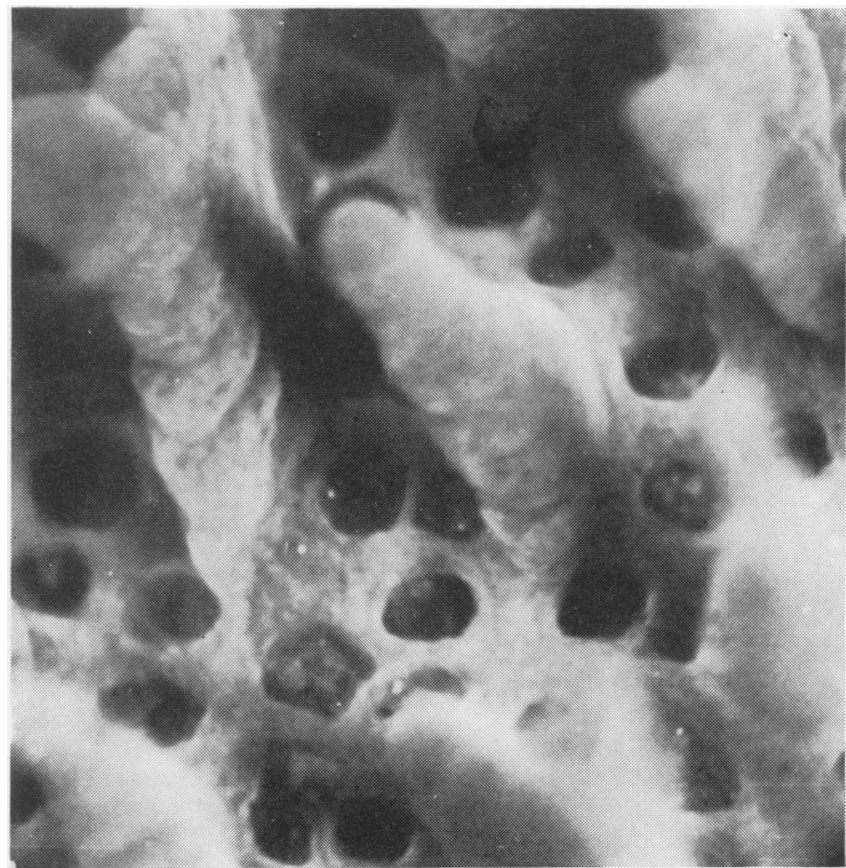

FIG. 2 . 


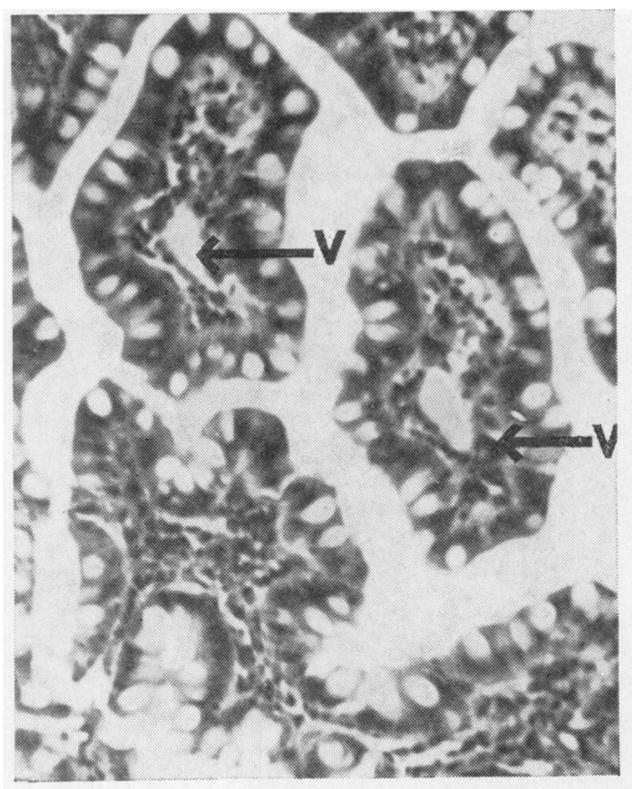

FIG. 3a.

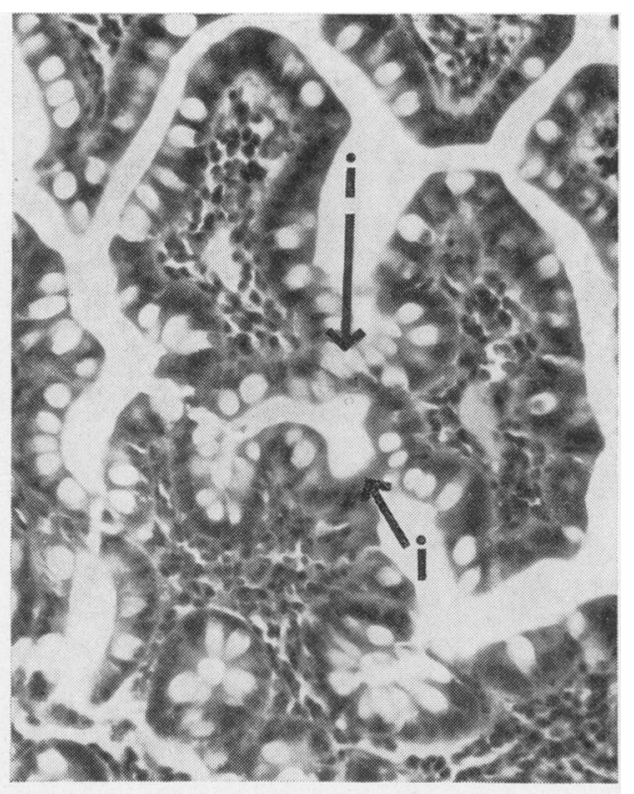

FIG. $3 b$.

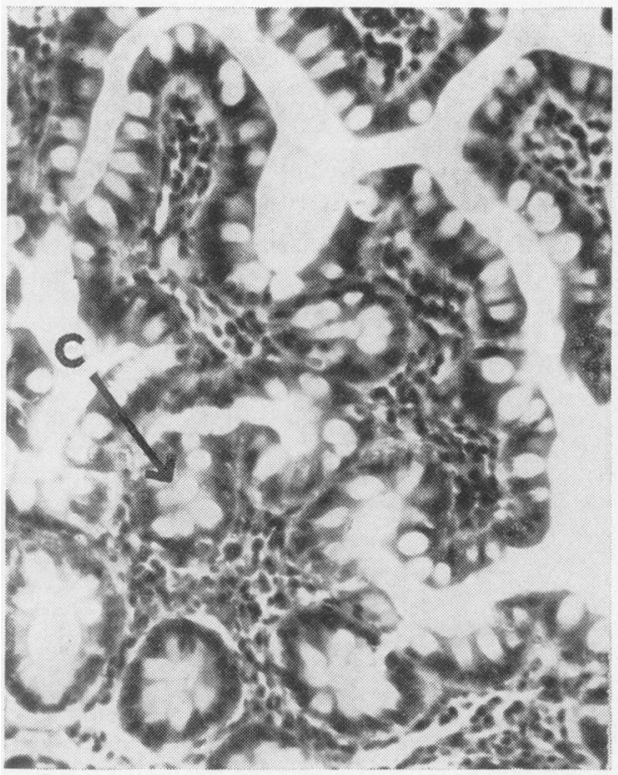

FIG. 3c.

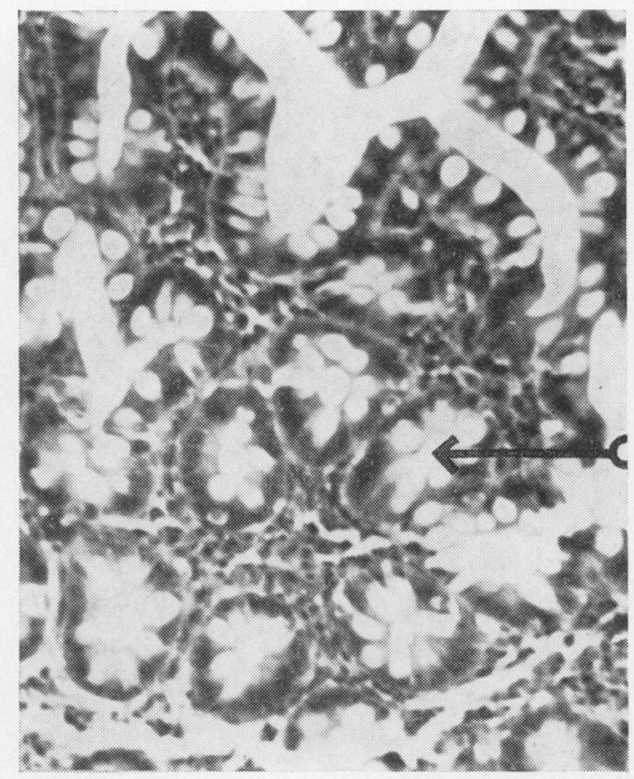

FIG. 3d.

FIG. 3. Serial horizontal cross sections through normal fixed ileum. Initially the section is through the base of three villi (a). The next section (b) demonstrates the delicate intervillous ridges, which already at the next level (c) give way to crypts. $\times 110$.

$v=$ villus. $\quad i=$ intervillous ridge. $\quad c=$ crypt . 
stage 5 , severely abnormal, characterized by a flat mucosa.

STAGE 1 (FIG. 2) In the autolysed mucosa crypts are easily visible and outnumber villi in the ratio of approximately three to one. In many cases there are two crypts between villi and the greater part of each crypt mouth is adjacent not to villi, but to other crypts. Clearly, therefore, the migration of epithelial cells from crypts to villi cannot be a simple direct progression. Running in between adjacent crypts and joining up onto villi are many small delicate structures that we have termed 'intervillous ridges'. These are extremely small and delicate in the normal jejunum and are best seen in the serial horizontal sections (Fig. 3). By this technique the delicate intervillous ridges present at this stage are seen joining the base of villi. This appearance of the normal jejunum and ileum, with crypts outnumbering villi, and small delicate intervillous ridges is shown as a diagram in Figure 8 (1).

STAGE 2 (FIG. 4) This stage represents the typical appearance seen in the normal duodenum. Many of the crypts appear to be lying in groups of two to four in 'pits' beneath the surface. Seen directly under the dissecting microscope it is clear that this appearance is due to hypertrophy of many of the delicate intervillous ridges, which here have become fuller and broader, giving, in the stained specimens, the appearance seen in Figure 4. The structures present at this stage are shown in a diagram in Figure 8 (2).

STAGE 3 (FIG. 5) The appearances at this stage were seen in several of the specimens examined. Here more intervillous ridges have hypertrophied, and villi have become correspondingly shorter and wider. In unautolysed material only the tops of the broadened villi and the occasional intervillous ridge would be visible at the surface to give the mucosa the typical appearance of leaves with occasional ridges. The progression to this stage is shown in Figure 8 (3).

STAGE 4 (FIG. 6) Here the intervillous ridges are even broader and more prominent, and have incorporated all villous projections. Even in unautolysed material many of the larger ridges would be visible and give rise to a convoluted pattern. Figure 8 (4) is a diagram of the mucosa at this stage.

STAGE 5 (FIG. 7) This is a flat mucosa. Here more intervillous ridges have come up, broadened and flattened, and the surface of the mucosa is now totally made up of these hypertrophied structures. Obviously some of the intervillous ridges have enlarged more than others, and, in the flat mucosa,

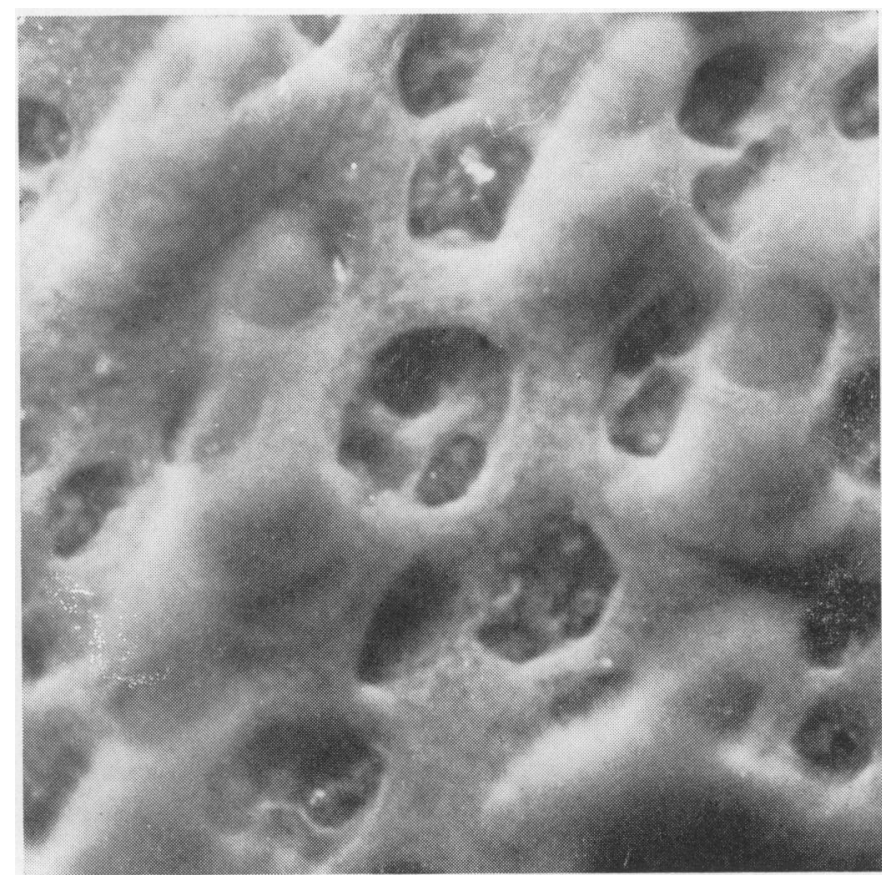

FIG. 4. Autolysed duodenal mucosa. Stage 2: here the intervillous ridges have begun to hypertrophy and the crypts are seen at their base. $\times 90$. 


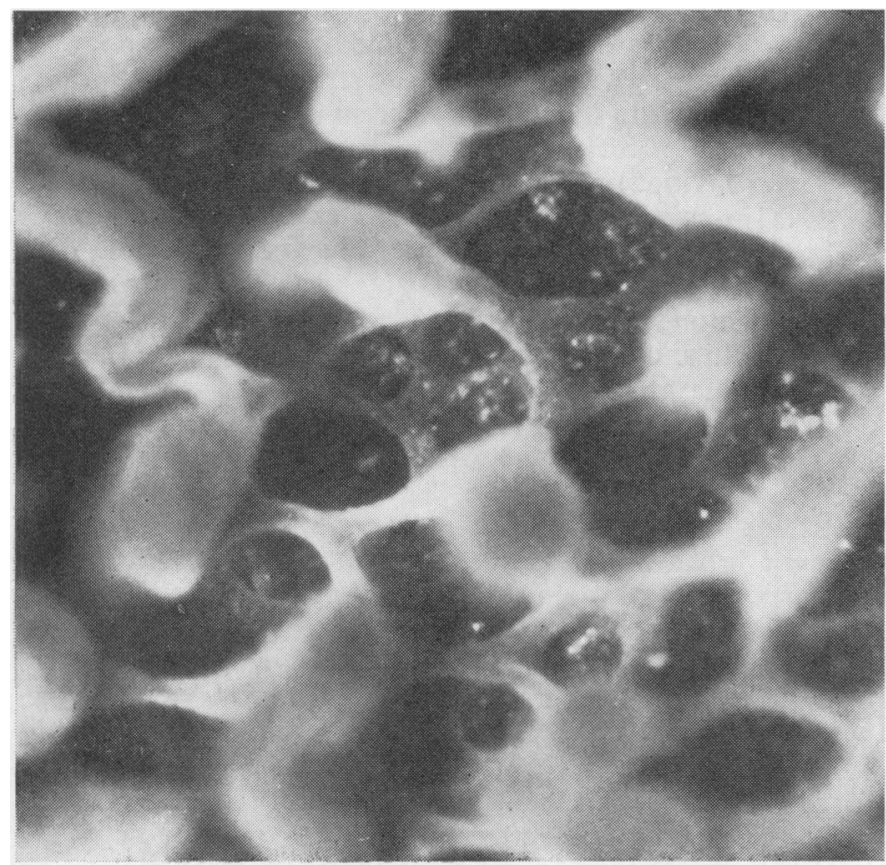

FIG. 5. Autolysed jejunal mucosa. Stage 3: there is further growth of intervillous ridges which in places are completely joining adjacent villi. $\times 90$.

FIG. 6. Autolysed jejunal mucosa. Stage 4: many intervillous ridges have further hypertrophied and are responsible for the convoluted patterns. $\times 90$.

FIG. 7. Autolysed jejunal mucosa. Stage 5: a flat mucosa totally made up of intervillous ridges. In places one or two crypt openings may be seen at the base of the ridges. $\times 90$.

FIG. 5.

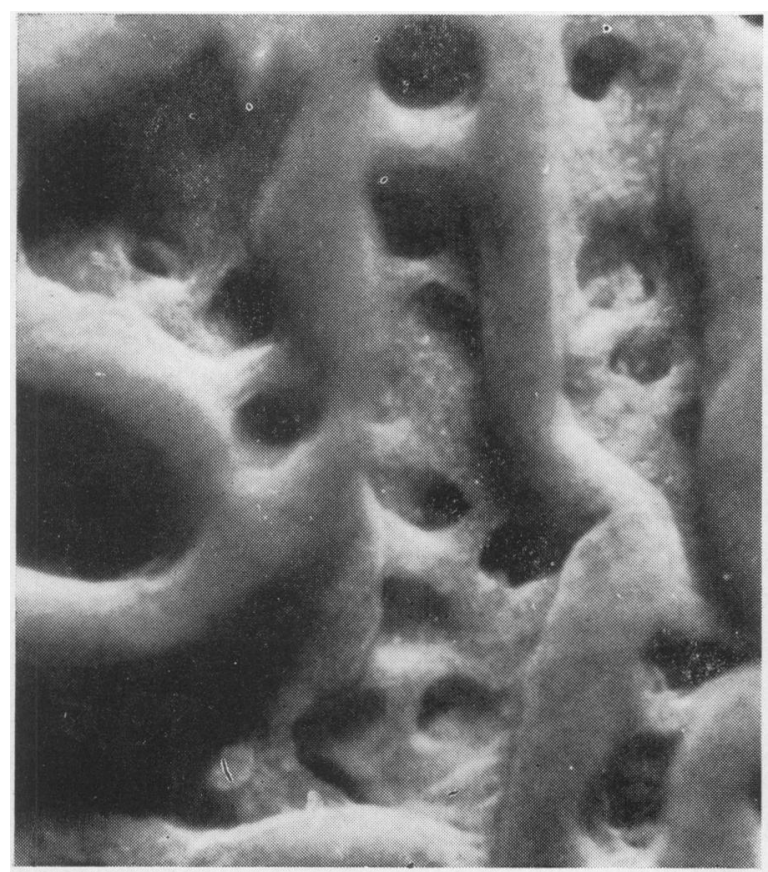

FIG. 6.

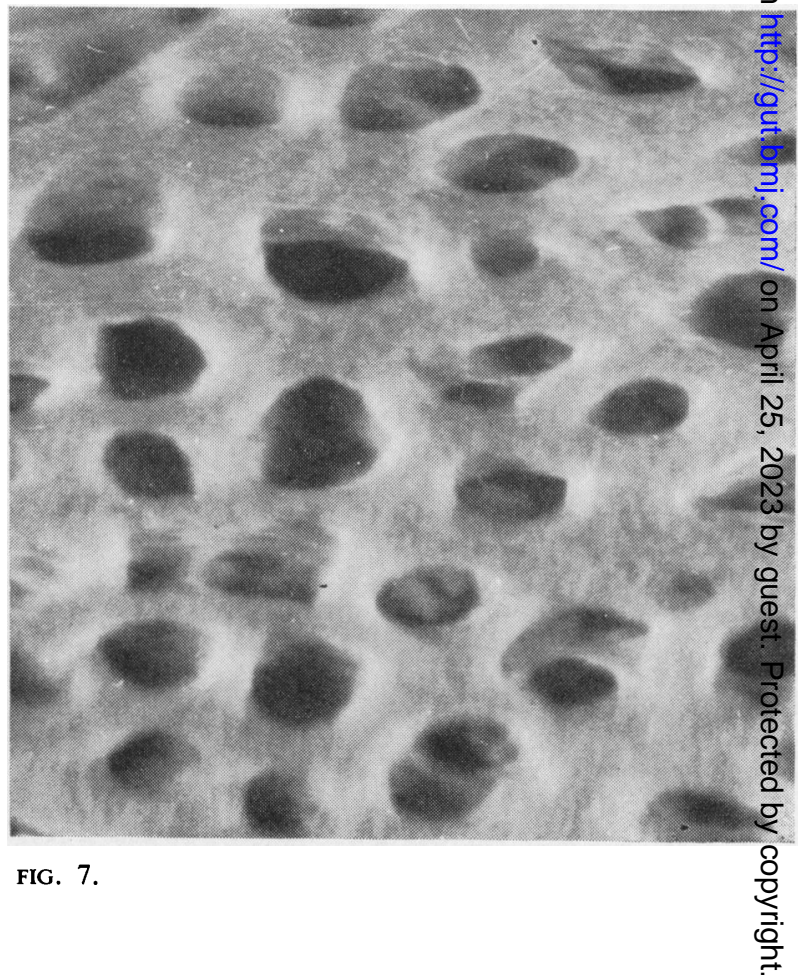



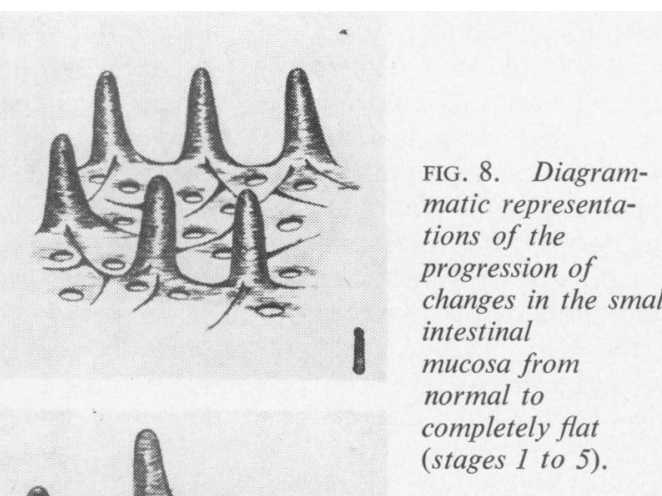

FIG. 8. Diagrammatic representations of the progression of intestinal mисоsa from normal to changes in the small
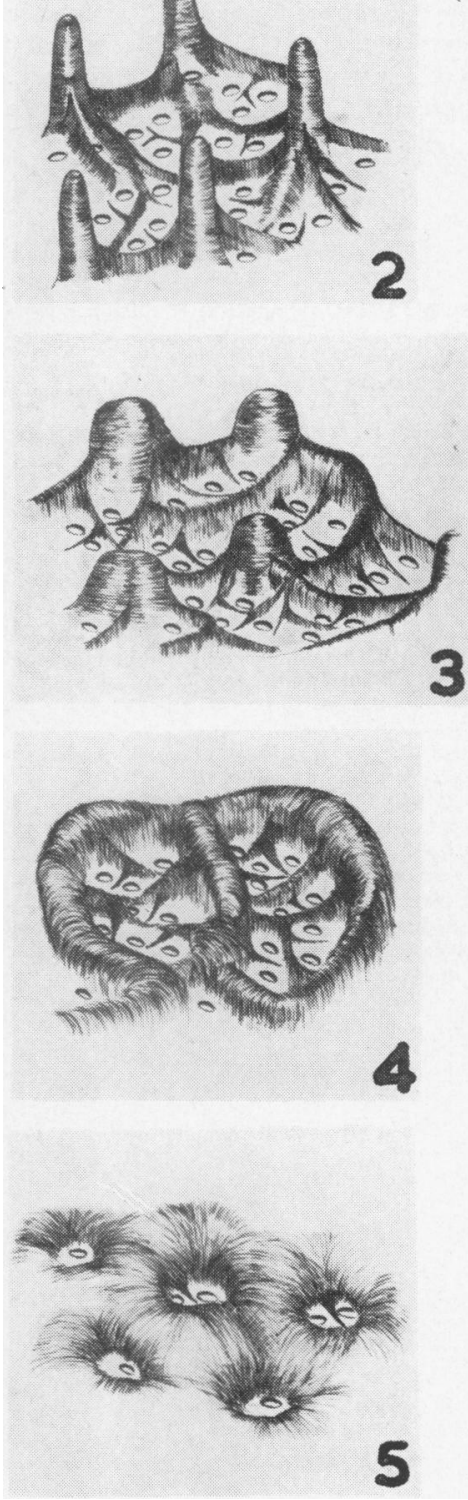

the broadest of these have fused together. These larger ridges are joined by smaller ones running between individual crypt openings (Fig. 8 (5)).

\section{DISCUSSION}

It is clear that the three-dimensional structure of the normal small intestinal mucosa is more complex than has been thought. Hitherto a simple one-to-one crypt-villus relationship has been assumed with alternate crypts and villi. However, direct study by the autolysis technique shows that crypts outnumber villi in the ratio of approximately three to one. This anatomical arrangement makes it impossible for many of the epithelial cells emerging from crypts to pass directly onto villi, and these must either be desquamated at the crypt mouth or else move round in some way to be channelled onto villi. A separate series of experiments has demonstrated that there is no widespread desquamation at the crypt mouth (Loehry, Croft, Singh, and Creamer, 1968), and it seems likely that the small intervillous ridges in the normal mucosa represent these 'migration lines' of the epithelial cells from crypts to villi.

In retrospect there is other evidence for this complex crypt-villus relationship. First, in histological sections, often more than one crypt is apparent between adjacent villi, and this has hitherto been considered due to a tangential section through the tissue. Secondly, in a study of cell kinetics in the human jejunum, Shorter, Moertel, Titus, and Reitemeier (1964) calculated a proliferation rate of crypt epithelial cells as one cell per 100 cells per hour. They also demonstrated that the average height of the crypt cell column was 30 cells, and it therefore follows that, in a two-dimensional plane, one crypt cell column produces one epithelial cell every three hours. They also calculated a rate for cell flow on the villi as one cell position per hour. From these data it follows that in a single villous cell column one cell will be lost from the tip each hour. However, the crypt cell column at the base of that villus will only produce one cell in replacement every three hours, and it could therefore be deduced that there must be three crypt cell columns supplying each villous cell column. The findings in the present study of three crypts to each villus by direct examination fits the mathematical data. Thirdly, by horizontal cross sections of three jejunal biopsies Cocco et al (1966) showed how two to four crypts opened out into a 'vestibule', and how these vestibules coalesced to form 'circumvillar basins', which then joined to form the 'intervillous space'. For ease in understanding the changes in these structures in disease, it is probably an advantage not to look upon this arrangement in these terms, but 
as a series of intervillous ridges of varying height, which in fact represent the walls of the vestibules and basins that Cocco et al describe. In the present study it is shown how the hypertrophy of these intervillous ridges provides the structural basis for the changes in mucosal pattern in disease. In the 'normal' duodenum the beginnings of this hypertrophy are already present, and it has been recognized for some time that minor variations in villous architecture are common in biopsies from this site. It is only, however, after autolysis that it can be seen how widespread are these minor changes in this area. As the mucosa becomes more abnormal more of the intervillous ridges hypertrophy even further as the villi shorten forming leaves, convolutions, and finally a flat mucosa where the whole mucosal structure is made up solely of hypertrophied and broadened intervillous ridges.

This hypertrophy, of course, does not occur equally at all sites at the same time, and explains why often a variety of villous shapes is evident in a single biopsy specimen, though, if the specimen is examined after autolysis, generalized intervillous ridge hypertrophy will be evident in even the most mildly abnormal mucosa. The crests of the mucosal folds show more severe changes than the troughs (Creamer, 1964), and with its extremely rapid turnover time the morphology of the mucosa must be in a state of continuous change, from one day to another and from one site to another, dependent upon multiple factors affecting each particular area of mucosa at any time. However, the characteristic changes in mucosal architecture depend ultimately upon variations of normal structures, and the increased prominence of normal epithelial cell migration lines is responsible for the changing patterns in disease states.

\section{SUMMARY}

The human small intestinal mucosa was examined under the dissecting microscope after autolysis of epithelial cells, and histologically by serial horizontal cross sections of conventionally fixed specimens. The normal three-dimensional mucosal structure was shown to be complex, with a preponderance of crypts over villi, and small intervillous ridges running between the crypts and joining up onto the base of villi. As the mucosa becomes abnormal these intervillous ridges hypertrophy to a varying degree as the villi shorten, and are responsible for the mucosal patterns that are seen in disease states.

We are indebted to Mr P. Leppard for technical assistance and to the Endowment Fund of St Thomas' Hospital for a grant to one of us (C.L.) while undertaking this work. This work formed part of an M.D. thesis (C.L.) at the University of Cambridge.

\section{REFERENCES}

Cocco, A. E., Dohrmann, M. J., and Hendrix, T. R. (1966). Reconstruction of normal jejunal biopsies: three-dimensional histology. Gastroenterology, 51, 24-31.

Creamer, B. (1964). Small-intestinal mucosal dynamics and the environment. Brit. med. J., 2, 1373-1375.

Himes, H. W., and Adlersberg, D. (1958). Pathologic changes in the small bowel in idiopathic sprue: biopsy and autopsy findings. Gastroenterology, 35, 142-154.

Loehry, C. A., and Creamer, B. (1966). Post-mortem study of smallintestinal mucosa. Brit. med. J., 1, 827-829.

—, Croft, D. N., Singh, A. K., and Creamer, B. (1968). Cell turnover in the rat small intestinal mucosa: an appraisal of cell loss. Gut, 10, 13-18.

Rubin, C. E., Brandborg, E. L., Phelps, P. C., and Taylor, H. C., Jr (1960). Studies of coeliac disease. I. The apparent identical and specific nature of the duodenal and proximal jejunal lesion in celiac disease and idiopathic sprue. Gastroenterology, 38, 28-49.

Shiner, M., and Doniach, I. (1960). Histopathologic studies in steatorrhoea. Gastroenterology, 38, 419-440.

Shorter, R. G., Moertel, C. G., Titus, J. L., and Reitemeier, R. J. (1964). Cell kinetics in the jejunum and rectum of man. Amer. J. dig. Dis., 9, 760-763.

Swanson, Y. L., and Thomassen, R. W. (1965). Pathology of the jejunal mucosa in tropical sprue. Amer. J. Path., 46, 511-551.

Townley, R. R. W., Cass, M. H., and Anderson, C. M. (1964). Small intestinal mucosal patterns of coeliac disease and idiopathic steatorrhoea seen in other situations. Gut, 5, 51-55. 